\title{
Express Screening of Tear Fluid for Evaluation of the Concentration of Lanomax Using Multisensory Stripping Voltammetry and Multivariate Statistics
}

\author{
I. I. Kolesnichenko ${ }^{1, *}$, V. A. Namiot ${ }^{3}$ and L. M. Balashova ${ }^{2,4}$ \\ ${ }^{1}$ Frumkin Institute of Physical Chemistry and Electrochemistry, Russian Academy of Sciences, RU- 119071, Moscow, Russia \\ ${ }^{2}$ Pirogov Russian National Research Medical University, RU-117997, Moscow, Russia \\ ${ }^{3}$ Lomonosov Moscow State University, Skobeltsyn Institute of Nuclear Physics, RU- 119991, Moscow, Russia \\ ${ }^{4}$ International Research and Practice Center for Tissue Proliferation, Non-commercial Partnership, Russia
}

\begin{abstract}
The drug concentrations monitoring is very important for finding the right dosage. The developed method is based on the recognition of multidimensional images, which are produced by multisensory electrochemical systems that provide reliable information on objects being tested, using multidimensional statistics. The measurements were carried out using a planar solid-state electrode. The test solution was a $0.05 \mathrm{M} \mathrm{KCl}$ solution, which contained the $\mathrm{Zn}_{2}{ }^{+}, \mathrm{Cd}_{2}{ }^{+}, \mathrm{Pb}_{2}{ }^{+}, \mathrm{Co}_{2}{ }^{+}$and $\mathrm{Hg}_{2}{ }^{+}$metal cations in the concentration of $5 \cdot 10^{-5} \mathrm{M}$. The efficiency of this method in determining lanosterol with accounting for changes in its concentration with over time has been demonstrated. In order to determine concentrations of Lanomax in tear fluid, a procedure for multisensory stripping voltammetry has been developed and tested. It has been shown that the effect of lacrimal fluid on metal dissolution currents in the test system lasts 12 hours after the Lanomax instillation.
\end{abstract}

\section{Introduction}

The constancy of the composition of blood is of particular importance for the organism. Its resilience has been studied in some detail concerning the active reaction $(\mathrm{Ph})$, the ratio of electrolytes, glucose, and number of the formal elements and others. Thanks to the intensive development of high-tech methods for research in the modern world, there are many new diagnostic capabilities. However, a large amount of information, which we expect to get, is often unavailable. This raises the question about meaningful etiopathogenic using these techniques, especially in ophthalmology, because now there is no simple, informative and pathognomonic method for early diagnosis of the disease. Data for the study of the effectiveness of these techniques are not so much, informative of them at different ophthalmic pathology practically not studied, and this makes it difficult to determine their role in the survey. While medical and social importance of such pathology as glaucoma, myopia, retinopathy and AMD (agerelated maculardegeneration) is so serious and indisputable that the relevance of the research that the actuality of the early detection of these diseases and their treatment becomes the No. 1 problem at the State Level. Despite the numerous methods of diagnosis and methods of treatment of the problem of blindness and a high level of disability from this ophthalmic pathology cannot be solved for many reasons. This and later identification of the disease and asymptomatic period of development, the large labour input surveys and high cost. We offer an innovative method that does not require expensive and time, easy to use and does not require the patient to any effort as objects of research are assumed biological fluids (blood, urine, tear, etc.) or tissues taken during the operation. A study carried out for the first time in ophthalmology. As a method of study are invited to use multisensory inversional (thick-film electrode) voltamperometry (IVA) to determine the status of the homeostasis of the organism as a whole, assessing whole blood or serum and local homeostasis, appreciating the tear fluid of the anterior

Chamber of the eye and the vitreous. This allows you to trace the evolution of biological fluids organism depending on the evolution of the pathological process. Built because of express-diagnostics of biological fluids will be used for the preliminary assessment of various operational states of the organism. A method for recognition of multidimensional images derived from analysis of multisensory voltamperometry electrochemical test system in the format of "language. Fundamental scientific problem is to define mechanisms for the development of systemic and local changes in ophthalmopathology.

Effective diagnostics through early disease detection implies simple procedures for initial patient examination, thereby reducing time and labor costs. In the line of the concept of metabolic profile, clinical chemistry is mostly concerned with analysis of body fluids. When conducting a comprehensive analysis of clinical chemistry is mainly limited to studies of the composition of the fluids of the

e-mail: kolesnichenko-ii@mail.ru 
human body [1]. A fundamentally new approach to the analysis of volatile compounds associated with the use of devices "electronic nose" [2]-[4]. When using the device "electronic nose" and "electronic tongue" [5] each analyzed substance corresponds to some characteristic pattern of feedback system that enables multidimensional discriminate against various substances (or mixtures of substances) by comparing them to images formed in advance N-dimensional image database [6]. A fairly complete list of models "electronic" nose and electronic tongue" is presented in [7],[8]. A number of foreign and domestic firms made bids for Annex developed analytical tools for evaluating the quality of foodstuffs [9] Wednesday, environmental monitoring [10], as well as for use in medical diagnosis [11]

The effective dosage of a new ophthalmic medicine is determined from the concentration-time profile of the drug in lacrimal fluid. Many analytical problems are solved by electrochemical methods which are quite simple, quick, and effective techniques for reliable quantitative analysis. The method of multisensory stripping voltammetry (MSV) for analysis of organic substances was developed at the Frumkin Institute of Physical Chemistry and Electrochemistry, Russian Academy of Sciences. This method is used to detect changes in the electrochemical activity of metal cations in solutions; the changes are due to their interaction with organic substances [13-19]. Metal cations (components of the test system) that are able to form complex compounds with organic substances are introduced into a 0.05 solution of $\mathrm{KCl}$. The introduction of these metal cations is accompanied by peak dissolution currents on voltammograms: metal cations interact with organic compounds and resulting changes in metal dissolution currents are reflected on stripping voltammograms. These changes are evaluated either individually for each metal of the test system or integrally. Measurements are made on one indicator electrode, which has a definite advantage over those multisensor methods that use a set of electrodes. The method involves using a pre-created database to identify organic substances. It proved to be effective when determining the concentration of ophthalmic betaxolol [21,22]. in tear fluid. It was determined using a calibration function. We used the MSV method to build the concentration-time profile of betaxolol dissolved in tear fluid. It was shown that the concentration of betaxolol in tear fluid remains constant for 12 hours after instillation. not only via eye drops, but also through injections directly into the vitreous body of the eyes. The results of experiments on human lenses extracted during cataract surgery turned out to be not that impressive: the lanosterol solution was apparently unable to deliver lanosterol molecules into lenticular opacities [23]. Nevertheless, like many foreign authors, we believe that if lanosterol is combined with some drug delivery system, lanosterol can be possibly transferred directly into the crystalline lens. Lanomax produced in the USA is a drug administered via eye drops to animals. The active ingredient of Lanomax is lanosterol. Accordingly, no injections are required: Lanomax is administered directly in the form of eye drops. In our study we used the method of multicensory stripping voltammetry to determine how long lanosterol (as part of Lanomax) stays in the conjunctival sac.

Cataract is a fairly common eye disease. Medical treatment of cataracts is currently based on eye drops such as Quinax Oftan Catachrom, Taufone, etc. However, these medicines do not ensure resorption of opacities, just slowing down the further development of cataracts. K. Zhang, Professor of Ophthalmology at the University of California suggested [23]. Curing cataracts with eye drops, without recourse to surgery. The idea was instigated by the findings that the genome of children suffering from severe cataracts contains a gene mutation that interferes the production of lanosterol, a naturally occurring steroid in the body. Based on the findings, it was decided to test whether lanosterol has the ability to prevent or even eliminate cataracts. Indeed, experiments on rabbits and dogs produced impressive results: transparency was significantly improved in the lens of the animals. However, lanosterol is practically insoluble in water therefore the lanosterol solution was administered.

Cataract is a fairly common eye disease. Medical treatment of cataracts is currently based on eye drops such as Quinax Oftan Catachrom, Taufone, etc. However, these medicines do not ensure resorption of opacities, just slowing down the further development of cataracts. K. Zhang, Professor of Ophthalmology at the University of California suggested [23]. curing cataracts with eye drops, without recourse to surgery. The idea was instigated by the findings that the genome of children suffering from severe cataracts contains a gen utation that interferes the production of lanosterol, a naturally ccurring steroid in the body. Based on the findings, it was decided to test whether lanosterol has the ability to prevent or even eliminate cataracts. Indeed, experiments on rabbits and dogs produced impressive results: transparency was significantly improved in the lens of the animals. However, lanosterol is practically insoluble in water therefore the lanosterol solution was administered not only via eye drops, but also through injections directly into the vitreous body of the eyes. The results of experiments on human lenses extracted during cataract surgery turned out to be not that impressive: the lanosterol solution was apparently unable to deliver lanosterol molecules into lenticular opacities [23]. Nevertheless, like many foreign authors, we believe that if lanosterol is combined with some drug delivery system, lanosterol can be possibly transferred directly into the crystalline lens. Lanomax produced in the USA is a drug administered via eye drops to animals. The active ingredient of Lanomax is lanosterol. Accordingly, no injections are required: Lanomax is administered directly in the form of eye drops. In our study we used the method of multicensory stripping voltammetry to determine how long lanosterol (as part of Lanomax) stays in the conjunctival sac.

\section{Materials and methods}

The measurements have been done using the electrochemical multisensory analyzer developed at IPCE 
RAS (Moscow). The Analyzer consists of two blocks, connected by a USB cable (Fig. 1):

- PC (laptop) with installed software, manipulator and USB cable;

- Measurement unit, consisting of electronic signal processing block, replacement electrodes and disposable

electrodes.

On the lid there are three LEDs to indicate the respective electrochemical cells and to indicate the connectionwith the PC. Based on are threaded and socket connector for thereplacement electrodes. On one of the sides ofthe hull removed the USB connector.

The replacement electrodes is a card with the connector for the connection of electronic signal processing block and three connectors to install disposable, replaceable electrodes.The replacement electrodes is a card with the connector for the connection of electronic signal processing block and three connectors to install disposable, replaceable electrodes.

The analyzer embodies a new approach using a solution containing various metal ions as an electrochemical test-system, the ions being able interact with organic substances to form complex compounds [21]-[23]. All known E-tongue devices use a set of electrodes as sensors, therefore the reproducibility of characteristics of the multi-sensor systems is problematic, which affects the reliability and pattern recognition in a negative way. We propose enhancing the reliability and pattern recognition of our multi-sensor stripping voltamperometry method through the use of metal cations, dissolved in the background electrolyte, as sensors. One electrode is used to record voltamperograms by the stripping voltamperometry technique to obtain multidimensional patterns of analytes.

The test-system (background electrolyte) is a $0.05 \mathrm{M}$ $\mathrm{KCl}$ solution containing the metal cations such as $\mathrm{Zn} 2+, \mathrm{Cd} 2+, \mathrm{Pb} 2+, \mathrm{Cu} 2+, \mathrm{Co} 3+, \mathrm{Hg} 2+$ as sensors. Thus an array of sensors is formed on one electrode allowing the deviceto be used as both E-tongue. The measurements have been done using planar electrodes (Autocom, Moscow), which represent polypropylene plates with working and auxiliary electrodes (carbon material) and silver-chloride reference electrode (Brown electrode) The voltamperograms (sensor outputs) recorded by the method of stripping voltamperometry are characterized by specific features (Fig.1) and good reproducibility.

The appearance of the voltamperograms change when different organic substances are added to the test-system. Each of the organic substances (or mixture of substances) produces specific changes, which allows their discrimination. The experimental study used the serum samples provided by Pirogov Russian National Research Medical University in accordance with a scientific cooperation agreement. The test-system solution, as background electrolyte, also serves as a concentratorfor volatile impurities.

Measurements were made on a planar electrode (1, 2). The test system solution consisting of $0.05 \mathrm{M} \mathrm{KCl}$ contained cations of metals $\mathrm{Zn} 2+, \mathrm{Cd} 2+, \mathrm{Pb} 2+, \mathrm{Co} 2+$, and $\mathrm{Hg} 2+$ in a concentration of $5 \cdot 10-5 \mathrm{M}$. First, the test system solution was applied to the indicator electrode and a background stripping voltammogram was obtained. The potential of cathodic metal deposition was set to $-1.55 \mathrm{~V}$ (with respect to the silver chloride electrode), which was followed by a potential sweep of up to $0.3 \mathrm{~V}$ (Fig. 1, curve 1).

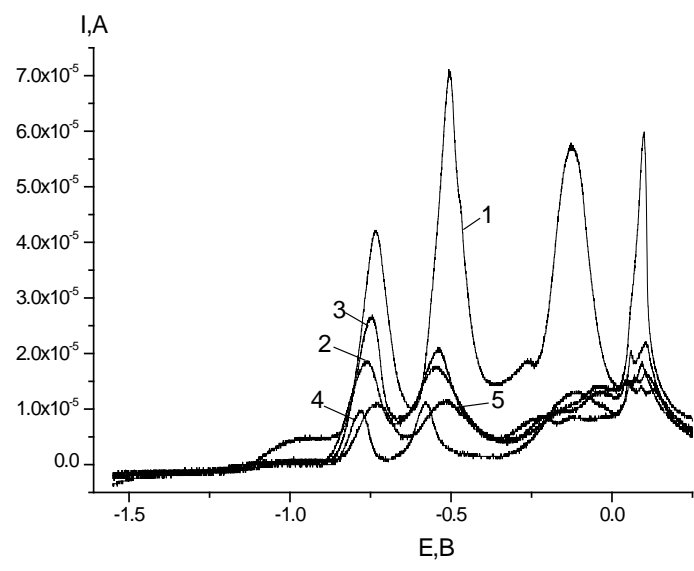

Fig.1. Stripping voltammograms of test system with Lanomax solutions of different concentrations: (1) test system; (2) $1 \cdot 10^{-7}$ $0.25 \%$ Lanomax; (3) $2 \cdot 10^{-7} \quad 0.25 \%$ Lanomax; (4) $3 \cdot 10^{-7}$ $0.25 \%$ Lanomax; and (5) $4 \cdot 10^{-7} 0.25 \%$ Lanomax.

Then, filter paper disks saturated with the Lanomax solution of different concentrations were successively placed on this electrode and stripping voltammograms were recorded and compared to the background voltammogram. Figure 1 shows how the stripping voltammogram changes with the concentration of Lanomax (curves 2 to 5).

Dissolution current peaks of the electrodeposited metals ( $\mathrm{Zn}, \mathrm{Cd}, \mathrm{Pb}, \mathrm{Co}$, and $\mathrm{Hg}$ ) are observed at the following potentials, respectively: $-0.99 \mathrm{~V},-0.75 \mathrm{~V}$, $-0.58 \mathrm{~V},-0.16 \mathrm{~V}$, and $0.09 \mathrm{~V}$. Therefore, Lanomax interacts with the metals of the test system, which makes it possible to detect Lanomax in tear fluid by the MSV method. This can be done by evaluating the integral change in the current plotted on the voltammogram.

\section{The effect of tear fluid on the test system}

We studied how tear fluid affects the metals of the test system. Tear fluid samples were taken from 10 healthy people. A disk of pre-treated filter paper, $8 \mathrm{~mm}$ in diameter, was placed in the conjunctival sac. Then, saturated with tear fluid, it was placed on the electrode with the test system solution and a stripping voltammogram was recorded.

All tear fluid samples affected the metals of the test system in a similar way (data spread of 12\%). Figure 2 shows the averaged effect of these samples on the metal dissolution currents (curve 2). The dissolution currents of metals decrease 4-7 times. This is due to the fact that organic substances of tear fluid are complexing agents. 


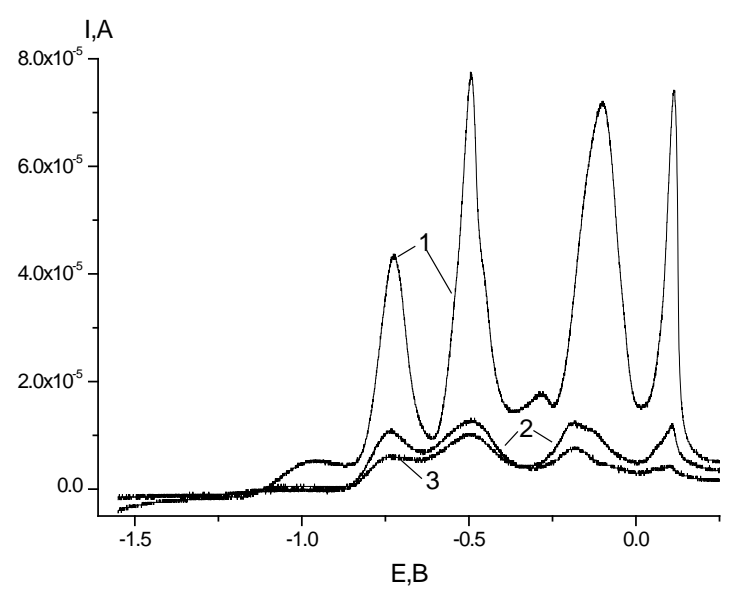

Fig. 2. Stripping voltammograms: Effect of tear fluid on the test system. (1) test system, (2) tear fluid, and (3) tear fluid after instillation of Lanomax.

\section{Statistical Data Processing}

\subsection{A Voltamperogram Is a Multidimensional Image of Primary Data}

The result of the operation is voltamperogram (Fig 2)current dependence of electrode potential, changing on linear time (sweep rate capacity). The peaks correspond to oxidation of metals test systems. Digital values (453 points) coming into the program for further processing in the form of a file from which to retrieve the value of the current. A voltamperogram is divided into $\mathrm{N}$-dimensional image, the amount of which depends on the complexity of the investigated system. Thus formed $\mathrm{N}$-dimensional image object (Fig 3).

\subsection{Data Dimensionality Reduction}

The next step is to group processing correlated sensors into clusters by reducing the dimensionality of data with the subsequent allocation of $\mathrm{M}$ bulk of characterizing the cluster information. $\mathrm{M}$ varied to achieve maximum discriminatory ability.

There are many algorithms to reduce the dimensionality of the data, some of which were tested in the study:

factor analysis, principal component analysis, cluster analysis, Fourier transform [24].

\subsection{Discrimination Algorithm}

The purpose of discriminatory analysis is attributing the object under study to a certain group/class by its characteristics in an optimal way, minimizing expected losses and the probability of misclassification. Discriminatory analysis, as part of statistical multiparameter analysis, comprises methods for classification of multidimensional objects through the use of available training samples.
Training samples were obtained by measuring parameters of known analytes to be subsequently stored in the database.

A similarity measure is the square of the Mahalanobis distance, which shows how close the object is to the centroids of the groups of known samples in the space of canonical roots. The results of the discrimination are visually presented as a graph in the space of the first and second canonical roots, the known analytes being depicted as clouds of points surrounded by ellipses and the sample under study as a point.

Thus, the discriminatory analysis allows the objects under study to be classified by a set of group/class attributes (Fig. 6).

The values of current were recorded in the database, assigned to one of three groups: "ZDOR", "BOL", or "TEST", indicating presumably healthy patients, or glaucoma patients, or the test-system (reference sample), respectively.

Three independent measurements of the blood serum of each testee are stored in the database, too.

The statistics software package Statistica 6.0 was used for data processing.

\section{The time on the concentration of lanomax in tear fluid after the eye drop instillation in cataract patients}

Lanomax (one drop) was administered into the conjunctival sac, after which a disc (8 $\mathrm{mm}$ in diameter) of pretreated filter paper was placed there. After a certain time, the paper disk was removed from the conjunctival sac and placed on the electrode with the test system (50 $\mu \mathrm{l})$. Measurements were taken after $30 \mathrm{~min}, 1 \mathrm{~h}$, and then every hour for 12 hours. Within 12 hours, no changes were observed in the effect of Lanomax on the dissolution currents of the metals of the test system in tear fluid of cataract patients.

\section{Results and Discussion}

\subsection{E-Tongue Technique}

Fig. 2 shows the voltamperograms recorded during analyses of both the test-system solution 1) and the testsystem solution with patients' samples of tear fluid 2). All voltamperograms for the tear fluid samples of healthy persons are alike, being different quantatively. The tear fluid samples of 6 patients and 14 presumably healthy people were used to estimate the possibility of discrimination based on the E-tongue technique.

Each patient's sample was measured three times, the data averaged. A table of the data comprises 6 rows marked with "BOL" and 14 rows marked with "ZDOR". A total of 20 calculations were done, each having the grouping mark ("BOL" or "ZDOR") of the current sample nulled, thus rendering the sample "unknown" for discriminatory analysis.

Fig 3 shows the results of discriminatory analysis in the form of 2D distribution of the samples of presumably 
healthy people and glaucoma patients. The two groups of points are surrounded by ellipses with a confidence probability of 0.95 .

The results of statistical analysis show that 3 out of 6 tear fluid samples of presumably healthy people and 9 out of 14 tear fluid samples glaucoma patients are discriminated correctly, which amounts to $50 \%$ and $64 \%$, respectively

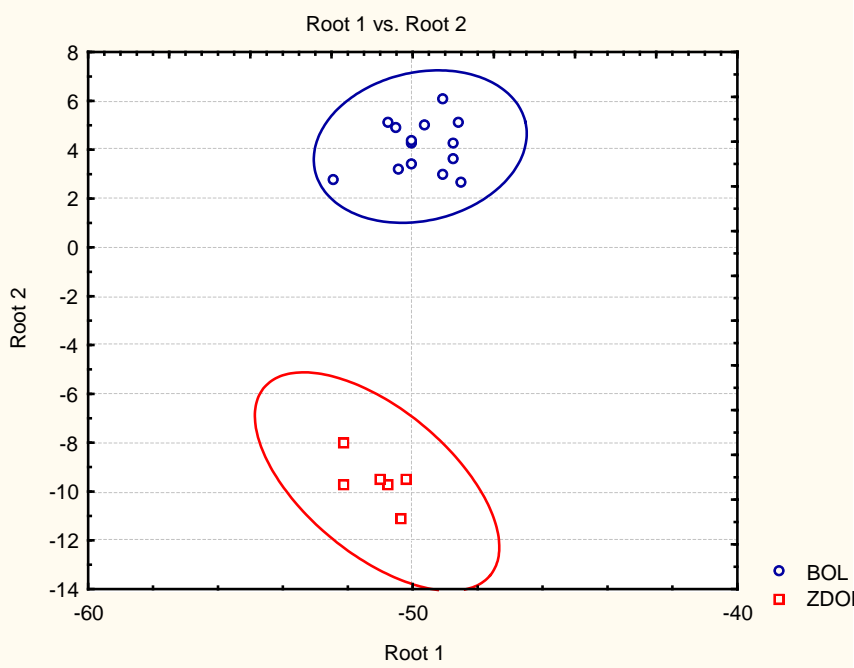

Fig. 3. Results of discriminatory analysis applied to E-tongue data. 2D distribution of patterns in the plane of the first and second canonical roots. The data dimensionality reduction technique is Fourier Transform. $M=20$.

\section{Conclusions}

It is shown that the MSV method can be used to detect Lanomax in tear fluid. It is found that the Lanomax concentration in tear fluid remains constant for 12 hours after its instillation in cataract patients. This is in agreement with the application instructions of Lanomax.

\section{References}

1. A. D’Amico, C. Di Natale, R. Paolesse, , et al., Sensors and ActuatorsB: Chemical, 130, 458-465. (2008) http://dx.doi.org/10.1016/j.snb.2007.09.044

2. D. Pickel, G. Manucy and D. Walker, Applied Animal. Behaviour Science, 89, 107-116. (2004). (2004) http://dx.doi.org/10.1016/j.applanim.2004.04.008

3. C.M. Willis, S.M. Church, C.M. Guest, et al., British Medical Journal, 329, $712 \quad$ (2004) http://dx.doi.org/10.1136/bmj.329.7468.712

4. M. McCulloch, T. Jezierski, M. Broffman, et al., Integrative Cancer Therapies, 5, 30-39. (2006) http://dx.doi.org/10.1177/1534735405285096

5. P. Pelosi and K.C. Persaud, In Sensors and Sensory Systems for Advanced Robotics. In: Dario, P., Ed., Springer-Verlag, Berlin, 361(1988)

6. S. Ampuero and J.O. Bosset, Sensors and Actuators B: Chemical, 94, 1-12. (2003) http://dx.doi.org/10.1016/S0925-4005(03)00321-6
7. D. James, S.M. Scott, Z. Ali and W.T. O’Hare, Microchimica Acta, 149, 1-17. (2005) http://dx.doi.org/10.1007/s00604-004-0291-6

8. N.V. Dolgopolov, and M. Yablokov, Peace and Security 3, 54(2007) [In Russian]

9. M. Aleixandre, J. Lozano, J. Gutirerrez, et al., Sensors and Actuators B: Chemical, 131, 7176(2008) http://dx.doi.org/10.1016/j.snb.2007.12.027

10. O. Helli, and M. Sia, Sensors and Actuators B, 103, 403-408 http://dx.doi.org/10.1016/j.snb.2004.04.069

11. E.R. Thaler, Laryngoscope, 112, 1533-1542. (2002) http://dx.doi.org/10.1097/00005537200209000-00002

12. D. James, S.M. Scott, Z. Ali, and W.T. O’Hare, MicrochimicaActa, 149, 1-17 (2005) http://dx.doi.org/10.1007/s00604-004-0291-6

13. N.V. Dolgopolov and M. Yablokov, Peace and Security 3, 54 (2007) [In Russian]

14. M. Aleixandre, J. Lozano, J. Gutirerrez, et al., Sensors and Actuators B: Chemical 131, 71-76 (2008) http://dx.doi.org/10.1016/j.snb.2007.12.027

15. O. Helli, and M. Sia, Sensors and Actuators B, 103, 403-408 http://dx.doi.org/10.1016/j.snb.2004.04.069

16. E.R. Thaler, Laryngoscope, 112, 1533-1542. (2002) http://dx.doi.org/10.1097/00005537-20020900000002

17. W.A. Groves, E.T. Zellers and G.C. Frye, Analytica Chimica Acta, 371, 131-143 (1998) http://dx.doi.org/10.1016/S0003-2670(98)00294-3

18. C. Di Natale, A. Macagnano, E. Martinelli, et al., Biosensors and Bioelectronics, 18, 1209-1218. (2003) http://dx.doi.org/10.1016/S09565663(03)00086-1

19. W. Cao, and Y. Duan, ClinicalChemistry, 52, 800811

(2006) http://dx.doi.org/10.1373/clinchem.2005.063545

20. V.P. Lukovtsev, A.N. Doronin, N.V. Lukovtseva, V.A. Semenov and V.M. Ganshin, Electrochemistry, 45, 869-872 (2009) [In Russian]

21. V.N. Andreev, V.M. Ganshin, A.N. Doronin, et al., Patent of the Russian Federation No.2375705 [In Russian]

22. I.I. Kolesnichenko, V.M. Ganshin, A.L. Kluev, et al., Proceeding of 4th International Scientific and Practical Conference. Publishing House of the Polytechnic University, Saint-Petersburg, 189 [In Russian]

23. L. Zhao, X.-J. Chen, J. Zhu, et al., Nature, 523, 607 (2015).

24. A.A. Khalafyan, Statistica 6. Statistical Data Analysis. 3rd Edition, Moscow. (2007) [In Russian] 